(mean age of onset $=5.21$ days). Imaging identified stroke in 6 cases, subrenal aorta thrombosis in one case. We identified one case of protein $\mathrm{S}$ defesciency, 4 cases of isolated factor $\mathrm{V}$ leiden mutation, one case of isolated hyperhomocysteinemia and one case of combined factor V Leiden and hyperhomocysteinemia. The last one was presented with multiple cerebral and abdominal thrombosis. Family screening was performed in 3 cases. Treatment was based on Fresh frozen plasma transfusion in newborn who had severe protein C deficiency. None of our patients was treated with thrombolysis. During followup, there was no recurrence of thrombotic events. Three patients had neurological deficit. Two newborns died of disseminated intravascular coagulation.

Conclusions Thrombotic disorders at an early age should lead to performing thrombophilia testing. Family screening is essential to detect asymptomatic deficiency. Clinical features and treatment depend on thrombosis localization and extension.

\section{P444 MANAGEMENT OF ABDOMINAL MASSES IN THE NEWBORN: EXPERIENCE OF THE NEONATOLOGY DEPARTMENT OF SFAX (TUNISIA)}

${ }^{1}$ Chiraz Regaieg*, ${ }^{1}$ Rim Zaghdoud, ${ }^{2}$ Hayet Zitouni, ${ }^{1}$ Amel Ben Hmed, ${ }^{1}$ Manel Charfi, ${ }^{1}$ Amira Bouraoui, ${ }^{1}$ Nedia Hmida, ${ }^{2}$ Riadh Mhiri, ${ }^{1}$ Afef Ben Thabet, ${ }^{1}$ Abdellatif Gargouri. ${ }^{1}$ Department of neonatology, Hedi Chaker Hospital, Sfax, Tunisia; ${ }^{2}$ Department of pediatric surgery, Sfax, Tunisia

\subsection{6/archdischild-2019-epa.780}

Background Abdominal masses in neonates reflect a wide spectrum of diseases, from lesions that can cause significant morbidity and mortality, to conditions readily corrected surgically, to entities which may be safely observed.

Objective To evaluate epidemiology, clinical features, management and outcome of abdominal masses in the newborn.

Methods It's a retrospective study of all cases of abdominal masses registered in the neonatology department of Sfax between 2004 and 2019 .

Results Thirteen patients were included in the study. A female predominance was noted (sex ratio $=0.18$ ). Antenatal diagnosis was made in 10 cases. Seven patients were born via cesarean section. The mean gestational age was 37.7 weeks. Mean birth weight was $3160 \mathrm{~g}$. Three patients had fetal acute suffering and respiratory distress. The most frequent physical finding was palpable abdominal mass $(n=9)$. Ultrasonography ( $\mathrm{n}=13)$, abdominal scan $(\mathrm{n}=3)$ and MRI $(\mathrm{n}=4)$ were used for diagnosis. Tumor sizes ranged from 4.6 to $10 \mathrm{~cm}$. We had identified renal cystic lymphangioma $(n=1)$, Infantile myofibromatosis $(n=1)$, ileal duplication $(n=3)$, hydrocolpos $(n=4)$ and ovrian cysts $(n=4)$. Total resection was the treatment for ileal duplication ,ovarian cysts and lymphangioma cysts cases. The newborn with infantile myofibromatosis received medical treatment (vincristine) after incomplete resection. The treatment of hyrocolpos was based on simple hymenotomy in two cases and laparotomy in the other two complicated cases. Mean follow-up time was 24 months. Only one patient who had giant hydrocolpos died of refractory shock and acute kidney failure 3 days after surgery.

Conclusions Most neonatal abdominal masses are due to benign lesions. Some of them may provide diagnostic difficulties. Most of masses require surgical treatment, which can be safely performed in small infants by trained personnel. However genuine controversy exists in the management of some lesions including infantile myofibromatosis.

\section{P445 \\ PLANNNG FOR THE NEW CHILDREN'S HOSPITAL: ANALYSIS OF PATIENT POPULATION AND RESOURCE USE IN THE NEONATAL UNIT OF TEMPLE STREET CHILDREN'S UNIVERSITY HOSPITAL}

1,25inead Brannick* ${ }^{3}$ Grace Crilly, $1,4,5 \mathrm{Naomi}$ McCallion. 'Temple Street Children's University Hospital, Dublin, Ireland; ${ }^{2}$ National Maternity Hospital, Dublin, Ireland; ${ }^{3}$ School of Medicine and Medical Science, University College Dublin, Dublin, Ireland; ${ }^{4}$ The Rotunda Hospital, Dublin, Ireland; ${ }^{5}$ School of Medicine, Royal College of Surgeons Ireland, Dublin, Ireland

\subsection{6/archdischild-2019-epa.781}

Aims The neonatal service of Temple Street Children's University Hospital (TSCUH) includes St. Michael's B ward (SMB; 8 beds) and the HDU (6 beds). Admissions to the HDU are restricted to transfers from maternity hospitals (Brennan \& Murphy, 2018). The new children's hospital ( $\mathrm{NCH}$ ) will establish a single entity to integrate the services currently provided by TSCUH, OLCHC, and the NCH Tallaght (Department of Health, 2017). The aim of this study is to produce qualitative analysis of the patient population/resource use in the neonatal $\mathrm{HDU} / \mathrm{SMB}$ in order to plan for delivery of care in the $\mathrm{NCH}$. Methods Using the nursing admissions books, data was retrospectively collected for 6 months of admissions (July to December 2017).

Results In the HDU (N=59), 15\% of patients had a CGA of $<37 / 40$. The median age was 5 days; the median LOS was 8 days (range: 1 - 125 days). $71 \%$ of patients were jointly admitted by Neonatology/surgical specialty. The most common diagnoses were myelomeningocoele (14\%) and TOF (12\%). $19 \%$ of patients required NIV; $27 \%$ received TPN.

In SMB $(\mathrm{N}=279), 2 \%$ of patients had a CGA of $<37 / 40$. The median age was 6 weeks; the median LOS was 2 days (range: 1 - 22 days). $71 \%$ of patients were admitted under the care of General Paediatrics. The most common diagnosis was bronchiolitis $(22 \%)$ with a clear seasonal trend observed. $9 \%$ of patients required NIV.

Conclusion The neonatal ward in TSCUH offers a unique service, allowing access to specialist care that is not available in the maternity hospitals, and providing a high level of support to term/premature newborns. It is clear that a specialised neonatal ward is necessary to safely care for this patient population, along with separation from the significant burden of infection that is present on high-turnover general wards. We recommend the inclusion of a similar unit in the $\mathrm{NCH}$.

\section{REFERENCES}

1. Brennan, K, Murphy, J. (2018, March 9). Admissions criteria St Michaels B High Dependency Unit. Dublin: Temple Street Children's University Hospital.

2. Department of Health (2017). The new children's hospital. Retrieved June 2, 2018, from https://health.gov.ie/future-health/reforming-our-hospitals/the-newchildrens-hospital/

\section{P446 ASSOCIATION OF MATERNAL HEMOGLOBIN LEVEL WITH GESTATIONAL AGE AND BIRTH WEIGHT OF BABIES}

\footnotetext{
1,2Manoj Kumar Chaudhary*, ${ }^{1}$ Nisha keshari Bhatta, ${ }^{1}$ Mohan Chandra Regmi, ${ }^{1}$ Rupa Singh,

${ }^{1}$ Paricha Upadhyay. ${ }^{1}$ BPKIHS, Dharan, Nepal; ${ }^{2}$ PKMMH, Karjanha, Nepal
}

\subsection{6/archdischild-2019-epa.782}

Background Two million people, that is over 30\% of world's population is anemic. In Nepal prevalence of anemia in pregnancy is $42 \%$ and low birth weight varies from 12 to 15 